\title{
The Rotary Storing-and-separating Device Based on Radio Frequency Identification Technology
}

\author{
Dongyang Shang ${ }^{1}$, Bo Tang $^{2}$, Limei Wu ${ }^{1}$,Ruofei $\operatorname{Han}^{3}$,Jiayu Tian ${ }^{4}$ and Bo Li ${ }^{1}$ \\ ${ }^{1}$ College of mechanical and electrical engineering,Shenyang Aerospace Shenyang Aerospace \\ University, Shenyang 110136, China. \\ ${ }^{2}$ Engineering Training Center,Shenyang Aerospace University,Shenyang 110136, China. \\ ${ }^{3}$ College of Foreign Studies, Liaoning University ,Shenyang 11000, China. \\ ${ }^{4}$ College of Aerospace Engineering,Shenyang Aerospace University,Shenyang 110136, China.
}

Keywords: RFID, express storing-and-separating, logistics network, CATIA software

\begin{abstract}
Nowadays, bar code recognition technology is widely used for separating expresses in the market. There are many problems that exists in express logistics industry such as low level of intelligence and low sorting efficiency. Aiming at the above problems, the paper proposed a rotary storing-and-separating device based on Radio Frequency Identification Technology (RFID). The device structural composition usage and the principle and structure of RFID are expounded. The three-dimensional (3D) model and its auxiliary structure of the device are designed by the CATIA software. In order to improve the intelligence level of logistics industry, the paper proposed a newly designed express logistics network which is based on the rotary storing-and-separating device and applies to RFID. The software and the hardware of the express identification system are designed. From the aspect of the system hardware, the electronic tag and the module of reader designed based on the RFID. The rotary storing-and-separating device based on RFID that proposed in the paper can do good to improve the intelligence level of logistics industry and the express sorting efficiency.
\end{abstract}

\section{Introduction}

The shopping pattern of consumers is changing day by day from store shopping to on-line shopping. It becomes necessary to take expresses for people in daily life. There are many express sorting devices that mainly include the paper wrote by LE Winslow and YC Chow ${ }^{[1]}$. The rotary storing-and-separating device is designed in the paper that combines the advantages of many types of sorting machines. It becomes a key issue in the development of express transportation industry that how to recognize the information safely, efficiently and intelligently. At the moment, there are mainly two ways for express identification. They are two dimensional bar code recognition technology and electronic tag based on Radio Frequency Identification (RFID) technology. It is hard to be popularized widely because of the high price of the electronic tag RFID technology ${ }^{[2]}$. Thus the bar code recognition technology is widely used in logistics industry.

Recent years, it can be found in the application of bar code recognition that it occurs frequently that the bar code cannot be recognized when it is damaged during transporting and sorting express.With the progress of large-scale integrated circuit technology and the constantly expanding production scale, the cost of production using RFID will continuously reduce and it will be widely used. Recent years, RFID has been successfully used in logistics industry to highly improve the efficiency of warehousing logistics, but the application of RFID is still in the primary stage at home ${ }^{[3]}$. The RFID has many advantages. It can make non-contract identification. It is easy to operate. It can adapt to harsh environment. It can identify multiple tags at high speed. So RFID is applied to logistics industry. It applies RFID in the storing-and-separating device in the paper, a rotary storing-and-separating device based on RFID is designed. 


\section{The Introduction of Rotary Storing-and-separating Device}

\subsection{The Structure of the Device}

The logistics industry is developing at a high speed currently. The space structure design of the rotary storing-and-separating device is multi-layer and each layer stores the expresses of similar size.The rotary positioning device can be divided into parts - the rotary temporary storing cabinet and the reducer gear train. The details of the rotary temporary storing cabinet are expounded. The reducing gear train is consisted of four gears which are placed under the large disk. The modulus of the gears is $2.5 \mathrm{~mm}$ and their number of teeth is $20,20,40,60$. All of them are master gear. The reduction radio of the gear train is six and the speed of the motor is $300 \mathrm{r} / \mathrm{min}$, so the speed of the output axis is $50 \mathrm{r} / \mathrm{min}$. The small ring which is placed between the gear and the axis is used to limit the movement of the gear by fixing the location of the small ring on the axis. The configuration design above can fix or limit the reduction gear train. The three-dimensional (3D) model of the rotary positioning device is designed by the CATIA Software, as shown in Figure1.

The express-withdrawing device consists of two parts: the restricting disk and the gear rack. The restricting disk is a structure that is used to limit the rotation of the large disk. The quick sorting of expresses can be achieved in the withdrawing device by reasonable design of space structure. The 3D model of the device is designed by the CATIA software, as shown in Figure2.

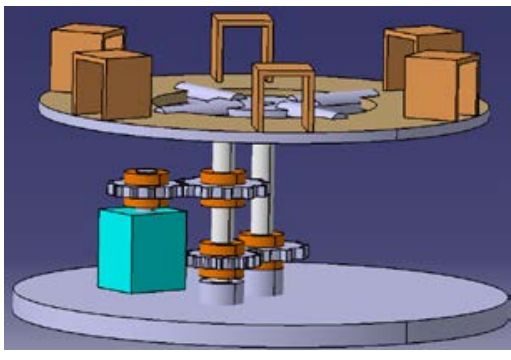

Fig.1. The stereo diagram of the rotary positioning device

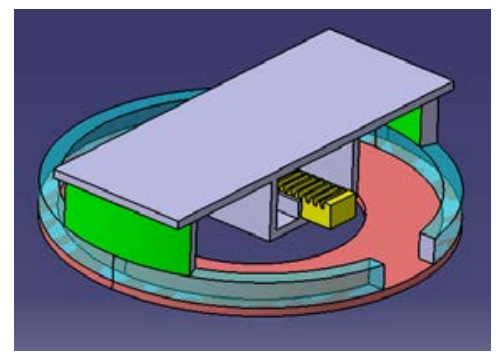

Fig.2.The stereo diagram of the express-withdrawing device

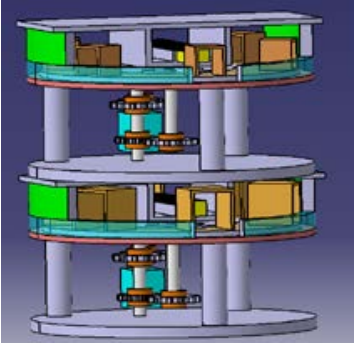

Fig.3.The stereo diagram of the rotary storing-and-separating device

\subsection{The Three- dimensional Design of the Device}

In the paper, the mathematics modeling (analysis hierarchy process) is used to obtain the optimized parameters of the temporary storage cabinet, and check the strength and rigidity of each gear of the designed reduction gear and get the strength and rigidity of each gear of the designed reduction gear, and get the appropriate gear parameters. After obtaining the above data, the part design of mechanical design part of the CATIA software is used to design the 3D structure of the remaining parts. The parts are assembled by the assembly design of mechanical design part of the CATIA software. The rotary storing-and-separating device is completed after assembling, as shown in Figure3.

The vehicle-mounted express translation auxiliary structure (vehicle translation structure) and the fixed-place express sorting auxiliary structure (the fixed auxiliary structure); the three-dimensional design of the auxiliary structure is shown in Figure 4. The vehicle-mounted express translation is designed by making a domestic trunk into a structure which is suitable for transporting things like large-scale or irregular shape of express shelves. The fixed-place express sorting auxiliary structure will be a certain size of room in which curved walls, electric, control system, vertical touch operation screen, alarm devices, camera devices are installed. The space structure of the room is designed reasonably to bring the consignee and the courier comfort when using the experience and the courier comfort when using the experience and achieve the automatic express storing and withdrawing functions. 


\subsection{Installation instructions}

The rotary express storing-and-separating device can be put into modular combination according to different structures to achieve sorting, transportation, storing, separation and other functions. The device can be wed singly by logistics sorting center to sort and store expresses. The device can be combined with the vehicle transportation structure to form a newly type of express transportation vehicle used in express transportation industry. The device can also be combined with the fixed auxiliary structure to form the terminal for consignees to receive expresses. Express storing and sorting can be unattended. It can be the terminal of communities and schools for automatically receive the expresses. The modular combination of the device is shown in Figure4.In this paper, the device is used alone named Model1:the logistics sorting-and-storing machine; the combination of the device and the vehicle transportation structure is named Model 2 :Express carrier ; the combination of the device and the fixed auxiliary structure is named Model 3 :the terminal for receiving expresses.

\section{Introduction to the Principle of RFID}

\subsection{Brief Introduction to RFID}

RFID is a new type of wireless communication technology and radio signal is used to identify specific targets and read-write relevant data. The technology is an automatic identification product which is first developed in 1970s.It was initially used by the US military, and recent years it becomes civilian product into the market ${ }^{[4]}$. This non-contact automatic identification technology can identify the target object and gain relevant data through radio-frequency signal. It doesn't need manual contact and optical visualization to input and deal the information. It is easy and fast to operate ${ }^{[5]}$. The RFID system generally consists of three parts. They are Tag, Reader and Antenna. The tag stores the information of the identified item and is usually placed on the surface of the recognized object. The reader uses the RFID to read-write the information of the tag. The RFID has been widely used in many areas in foreign countries, like expressway automatic charges traffic management, vehicle automatic identification and anti-theft, electronic tickets and so on. Based on the advantages of RFID, this paper applies it to the express storing-and-separating device in order to improve the efficiency and accuracy of express sorting.

\subsection{Introduction to the principle}

The basic working principle of the RFID system is introduced as follows. The electronic tag is an integrated circuit chip (IC chip).When electronic tags enter the effective working area, the antenna of the tag and the reader cut the magnetic induction line to produce the induced current. The reader's radio frequency carrier is used to provide energy for electronic tags. Then the electronic tags are activated. The way that the electronic tag transmits the stored data to the reader is load modulation ${ }^{[6]}$. The reader receives carrier signal of the electronic tag sent by the antenna and transmits the carrier signal to the computer background for processing. The processing system makes the logical judgment and analyzes its legitimacy through certain standard interface protocol, in order to issue the relevant instructions to make the system deal with it appropriately ${ }^{[7]}$.As the radio frequency identification technology is used in express sorting device, the required distance for identification is short, so the radio frequency identification system uses low frequency band. 


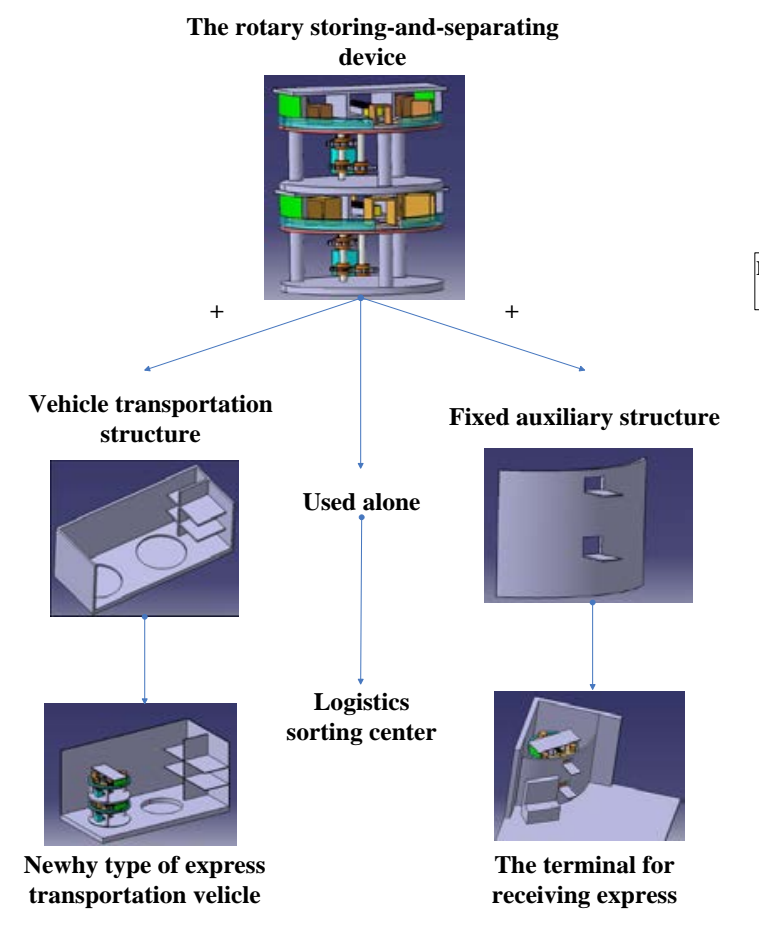

Fig.4. The Stereo Diagram of the Rotary Storing-and-separating Device

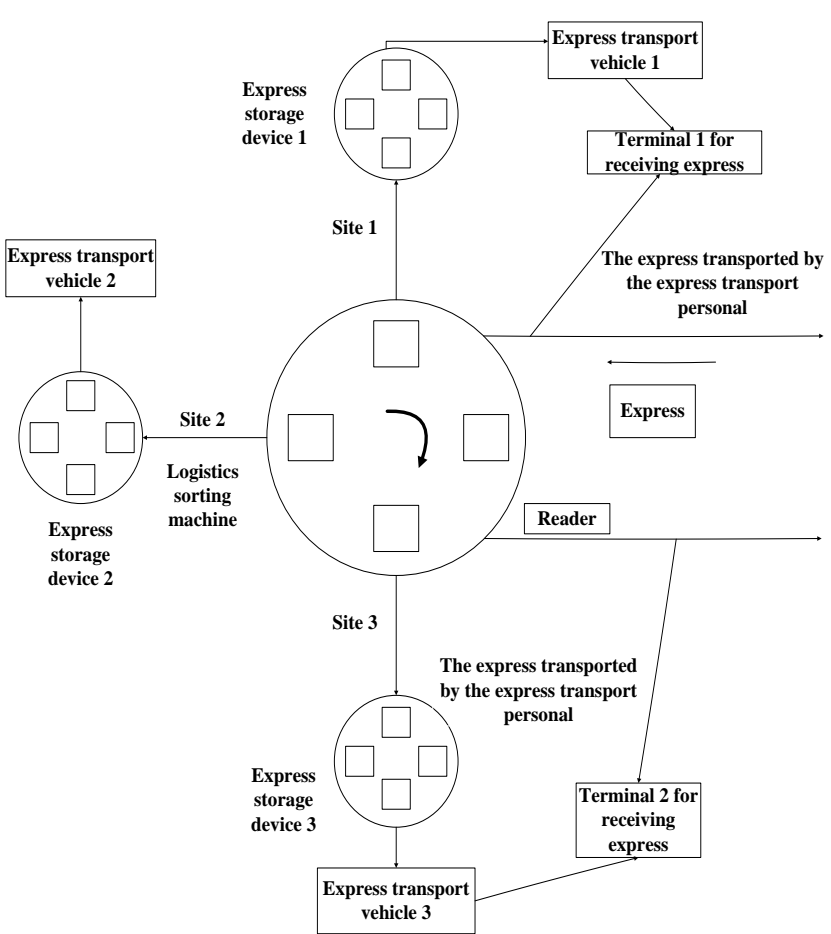

Fig.5.The schematic of the new type of logistics network

\section{Design of express information recognition system}

\subsection{Introduction to new logistics network based on radio frequency identification}

The RFID system designed in the paper is used in express sorting, storing and transportation industry. It can be seen from the above that the rotary storing-and separating device can choose different structure combination mode according to the needs. The mode 1 is logistics sorting machine. The mode 2 is express transport vehicle. The mode 3 is terminal for receiving express. These three modes can be applied to the RFID technology achieve their respective functions.

The RFID technology can be applied to the mode 1 . The RFID technology can be applied to the logistics sorting center to make express sort and store. The electronic tag is placed in the express and the reader is placed in front of the device. When the express enter the device, the information of the electronic tag is read and is compared to the background database. The region that the express belongs is judged, and then the device rotates to the corresponding region and release the express to realize the classification of the express. Similarly, according to the principle above, the express that need to be sent to the same region can be put in the same device to store.The RFID technology can be applied to mode2 to achieve real-time positioning of transport vehicles. The user can know the transport information of the express easily.The RFID technology can be applied to mode 3.It can be the unattended terminal for receiving express used in communities; schools and other logistics-intensive areas. There are two ways for storing the express. One is manual storage by the express transport personal. The other is an express transportation vehicle in the mode 2 which is used to realize the replacement of the device (i.e. replace the empty device with the device that is equipped with express).

The three modes can be combined into new type of logistics network with the RFID technology. In the network, the rotary express storing-and-separating device is regarded as the basis. The logistics sorting center is regarded as the center. The terminal for receiving express is regarded as the node. The intelligent network of logistics transportation is made through the express transport vehicle that connects the center and the node. The new type of logistics network that is based on the RFID technology is shown in Figure 5. 


\subsection{Design of System Hardware}

\subsubsection{Design of Tag}

The tag is placed on the surface of the express and is responsible for recording and storing the information of the express. The DPS-based tag is generally composed of modulator, code generator, clock, antenna and other components. The memories store the corresponding information of the express. The clock is the timing of the circuit function. This enables the data in the memory to be sent to the reader in proper order at the precise time. When the information of the express is read, the encoder encodes the information stored in the memory. The modulator receives the encoded information and sends it out through antenna. The tag also needs to be installed the voltage demodulator besides the above components. The voltage demodulator converts a portion of energy of the RFID signal into DC power in the way of inductive coupling. This can be more conducive for the tag to work. Functions of each module in the tag that used for express sorting are shown in the Figure 6.

\subsubsection{Design of the Reader}

The reader is placed at the terminal for receiving express to read the information stored in the tag. The reader is known as signal reception and control system which is generally composed of antenna, RF module, reading module. The RFID module which is mainly composed of the oscillator, the processor, the receiver, the amplifier and the power regulator is responsible for activating the tag and provide energy for the IC chip. The amplifier amplifies the RF carrier signal and sends it out by the antenna. The reading module is the core module of the reader which is responsible for controlling the reception and the delivery of information, and store the information of the tag. The reading model is composed of the processor, the amplifier, the decoder, the clock, the power and other components. The information stored in the tag is obtained after the decoder decodes. The reader is also installed the auxiliary display screen in order to make the operator to know the information. The structure of the reading module is shown in Figure 7.

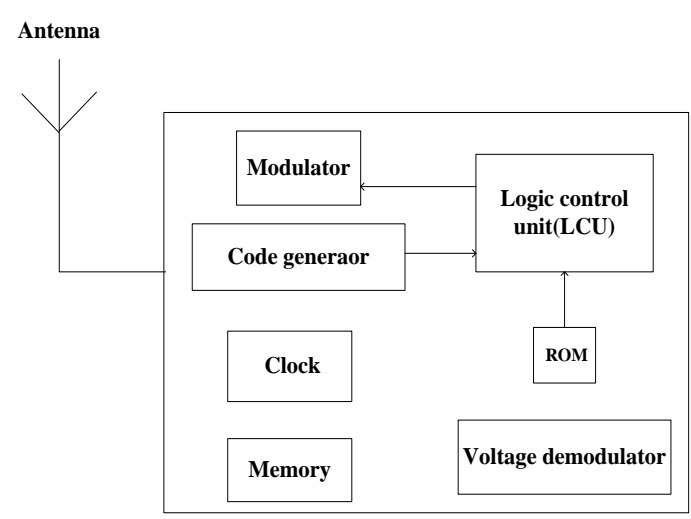

Fig.6.The schematic of the composition of tag

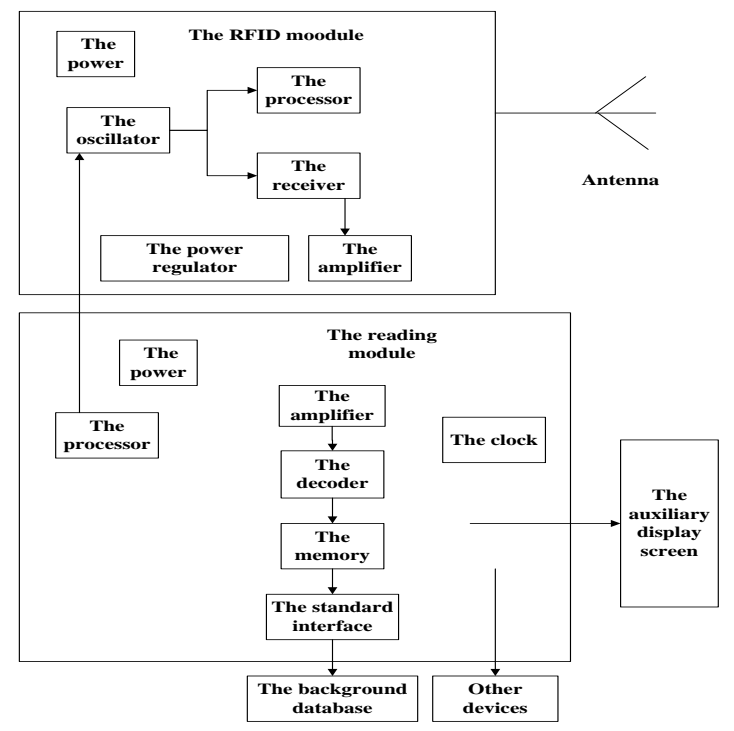

Fig.7.The Schematic of the Composition of the Reader

\section{Conclusion}

The rotary express storing-and-separating device proposed in the paper designs the express sorting system that applies the RFID system. The new type of intelligent logistics network is introduced in the paper. The combination of the express sorting device and the RFID technology can greatly improve the efficiency and the intelligence level of express sorting. The design proposal in the 
paper is of use value and promotion value. There are still the following problems need to solve and optimize in the study of the paper.

1). The use of ring-shaped temporary storage cabinet is convenient to manage the storage, but it causes a certain space waste.

2) PLC is used in the hardware of the system. It is not easy to find the fault point when there is a circuit dropout. The corresponding fault diagnosis circuit needs to be designed in the system. 3 The system should be encrypted. The system should be encrypted.The subsequent research on express sorting should be combined with the internet of things to achieve the entire intelligent sorting operations.

\section{Aacknowledgments}

This work is partly supported by College Students Creation and Career Training Project of Shenyang Aerospace University,NO.S1610605, and the National College Students Creation and Career Training Project in years of 2016, NO.201610143068.

\section{References}

[1] LE Winslow, YC Chow:IEEE Transactions on Computers (USA ,Washington, July 1983 ). Vol. 32, p.677-683.

[2] K.L.Wang, X.S.Liu: Journal of Luoyang Institute of Science and Technology (Natural Science Edition), Vol. 26 (2016) No.2, p.66-71.(in Chinese)

[3] X.H. Song, L.L. Chen, J.Z. Chen and P. Xia: Wireless Communication Technology, Vol. 19 (2016) No.2, p.52-56.(in Chinese)

[4] L.Z. Wang: China Safety Science Journal, Vol. 16 (2006) No.4, p.76-80.(in Chinese)

[5] X.H. Wang, X.G. Zhou: Modern Electronics Technology ,Vol.28(2005) No.11, p.30-33.(in Chinese)

[6] X.F. Shang, P.F. Xu: Modern Electronics Technology ,Vol.32(2009) No.19, p.29-31.(in Chinese)

[7] Z.L. Lu, H.F. Shen, J.Chen: Journal of Jiangxi University of Science and Technology ,Vol.32(2011) No.06, p.53-56.(in Chinese) 